Bull. Austral. Math. Soc.

VOL. 58 (1998) [271-290]

\title{
DISCRETE QUADRATIC ESTIMATES AND HOLOMORPHIC FUNCTIONAL CALCULI IN BANACH SPACES
}

\author{
EdWin Franks and Alan McIntosh
}

\begin{abstract}
We develop a discrete version of the weak quadratic estimates for operators of type $\omega$ explained by Cowling, Doust, McIntosh and Yagi, and show that analogous theorems hold. The method is direct and can be generalised to the case of finding necessary and sufficient conditions for an operator $T$ to have a bounded functional calculus on a domain which touches $\sigma(T)$ nontangentially at several points. For operators on $L^{p}, 1<p<\infty$, it follows that $T$ has a bounded functional calculus if and only if $T$ satisfies discrete quadratic estimates. Using this, one easily obtains Albrecht's extension to a joint functional calculus for several commuting operators. In Hilbert space the methods show that an operator with a bounded functional calculus has a uniformly bounded matricial functional calculus.

The basic idea is to take a dyadic decomposition of the boundary of a sector $S_{\nu}$. Then on the $k^{\text {th }}$ interval consider an orthonormal sequence of polynomials $\left\{e_{k, j}\right\}_{j=1}^{\infty}$. For $h \in H^{\infty}\left(S_{\nu}\right)$, estimates for the uniform norm of $h$ on a smaller sector $S_{\mu}$ are obtained from the coefficients $a_{k, j}=\left\langle h, e_{k, j}\right\rangle$. These estimates are then used to prove the theorems.
\end{abstract}

\section{INTRODUCTION}

Given a closed densely defined operator $T$ acting on a complex Banach space $\mathcal{X}$, and a function $f$ which is bounded and holomorphic on an open neighbourhood of the spectrum $\sigma(T)$ of $T$, the Riesz-Dunford functional calculus allows one to define a bounded operator $f(T)$. Bounds for many interesting functions of an operator may be obtained in this fashion. However, some functions, such as the imaginary powers and rational functions of the square root, may fail to be bounded and holomorphic on an open neighbourhood of the spectrum of $T$. Good methods for defining $f(T)$ exist whenever $f$ is bounded and holomorphic on an open set $\Omega$ such that the closure $\bar{\Omega}$ contains $\sigma(T)$, the boundary $\partial \Omega$ touches $\sigma(T)$ at finitely many points nontangentially, and $T$ has no point spectrum contained in this intersection, and has resolvent bounds near it. See $[13,2]$ for the case when $T$ is type $\omega$ and $\Omega$ is a sector.

Received 24th February, 1998

The authors received support from the Australian Government through the ARC.

Copyright Clearance Centre, Inc. Serial-fee code: 0004-9729/98 \$A2.00+0.00. 
In general $f(T)$ may be an unbounded operator [12]. If the norm of $f(T)$ is comparable to the supremum of $f$, then we say that $T$ admits a bounded $H^{\infty}(\Omega)$ functional calculus. Investigation of a necessary and sufficient condition for $T$ to admit a bounded $H^{\infty}(\Omega)$ functional calculus, namely that $T$ satisfies quadratic estimates, was begun by the second author in [13]. Subsequent investigations of quadratic estimates and related generalisations include $[2,5,7,9]$.

Of particular interest for this work is the paper [6] where among the many results, Cowling et al. show that weak quadratic estimates are equivalent to the boundedness of the holomorphic functional calculus for operators in a Banach space $\mathcal{X}$. They restrict their attention to operators $T$ with spectrum contained in the closure $\bar{S}_{\omega}$ of an open sector $S_{\omega}$ (defined below), and consider the question of whether $T$ admits a bounded $H^{\infty}\left(S_{\mu}\right)$ functional calculus for some $\mu>\omega$. Since $\bar{S}_{\mu}$ contains $\bar{S}_{\omega}$ and $\partial S_{\mu}$ touches $\bar{S}_{\omega}$ nontangentially at 0 and $\infty$ this question fits into the framework described above. The methods used in [6] to prove this result are quite technical, and their use of the Mellin transform would be less natural in more general settings. Specifically, they require some Fourier analysis to pass between continuous and discrete versions of the weak quadratic estimates. We avoid the use of Fourier analysis by directly formulating the problem in discrete terms. This gives simplified proofs of some of the results in [6] and allows an immediate extension to the more general situation outlined above.

Our techniques also generalise to several variables. For commuting operators on $L^{p}, 1<p<\infty$, which individually admit a joint functional calculus, one easily obtains Albrecht's extension to a joint functional calculus for several commuting operators. Uniform bounds on matricial functional calculi for operators in Hilbert space are shown, and used to prove similarity results.

Recently, Lancien, Lancien and Le Merdy have used this work to prove results about the boundedness of joint functional calculi on various types of Banach spaces, with applications to maximal regularity $[10,11]$.

We now give a brief outline of the paper. In Section 2 we attend to definitions and recall some required results in operator theory. In Section 3 we construct a sequence of functions $\left\{\Phi_{m, k, j}\right\} \subset H^{\infty}\left(S_{\nu}\right)$ which spans $H^{\infty}\left(S_{\nu}\right)$ and has the property that, if $\mu<\nu$, then any linear combination of the $\Phi_{m, k, j}$ 's with bounded coefficients is in $H^{\infty}\left(S_{\mu}\right)$. In Section 4 we use the $\Phi_{m, k, j}$ 's with an operator argument to provide some discrete quadratic estimates which an operator must satisfy in order to have a bounded $H^{\infty}\left(S_{\nu}\right)$ functional calculus. These discrete quadratic estimates easily generalise in $L^{p}, 1<p<\infty$, to the case of several commuting operators, and so provide a rather direct proof of the existence of a bounded joint functional calculus $[1,3]$. Section 5 extends the results of Sections 3 and 4 to more complicated domains. In Section 6 we use the results of Section 5 to show that on Hilbert spaces, the operators considered in Section 5 possess uniformly bounded matricial functional calculi, and apply this fact to a special case of the so-called 
"polynomially bounded" question of Halmos.

\section{Preliminaries}

Let us start with the case when the spectrum of $T$ is contained in a sector. The more general case is treated in Section 5. Accordingly, for $0<\nu<\pi$, let $S_{\nu}$ denote the open sector of angle $\nu$. That is, $S_{\nu}=\{z \in \mathbb{C}:|\arg z|<\nu\}$. Let $H^{\infty}\left(S_{\nu}\right)$ denote the algebra of bounded holomorphic functions on $S_{\nu}$, with norm

$$
\|h\|_{\infty}=\sup _{z \in S_{\nu}}|h(z)| \text {. }
$$

Throughout, $\mathcal{X}$ denotes a complex Banach space. By an operator in $\mathcal{X}$ we mean a linear mapping $T: \mathcal{D}(T) \rightarrow \mathcal{X}$, where the domain $\mathcal{D}(T)$ of $T$ is a linear subspace of $\mathcal{X}$. The norm $\|\cdot\|$ of $T$ is the (possibly infinite) number

$$
\|T\|=\sup \{\|T(u)\|: u \in \mathcal{D}(T),\|u\|=1\} .
$$

We say that $T$ is bounded if $\|T\|<\infty$, and write $T \in \mathcal{L}(\mathcal{X})$ when $T$ is bounded and $\mathcal{D}(T)=\mathcal{X}$. We call $T$ closed if its graph $\{(u, T u): u \in \mathcal{D}(T)\}$ is a closed subspace of $\mathcal{X} \times \mathcal{X}$. The spectrum and resolvent set of $T$ are denoted by $\sigma(T)$ and $\rho(T)$ respectively. The former set is the complement of the latter, which is the set of all complex $\lambda$ for which there exists a bounded operator, called the resolvent and denoted $(\lambda-T)^{-1}$, such that $(\lambda I-T)(\lambda-T)^{-1}$ is the identity operator $I$, and $(\lambda-T)^{-1}(\lambda I-T)$ is the identity operator on $\mathcal{D}(T)$.

DEFINITION 2.1. An operator $T$ acting on $\mathcal{X}$ is said to be of type $\omega$, where $0<\omega<\pi$, if $T$ is closed, $\sigma(T)$ is contained in the closure of $S_{\omega}$, and for each $\mu$ in $(\omega, \pi)$,

$$
\left\|(T-z I)^{-1}\right\| \leqslant c|z|^{-1}, \quad z \in \mathbb{C} \backslash S_{\mu} .
$$

An operator $T$ of type $\omega$ always possesses an $H^{\infty}$ functional calculus $[13,2,6,4]$. That is, for $\mu>\omega$ there exists a unique algebra homomorphism from $H^{\infty}\left(S_{\mu}\right)$ into the space of closed operators on $\mathcal{X}$ which takes $(\lambda-z)^{-1}$ to $(\lambda-T)^{-1}$. However, it may happen that $\|h(T)\|=\infty$, for some $h \in H^{\infty}\left(S_{\mu}\right)$ with $\|h\|_{\infty}=1[12]$. Of course if $h \in H^{\infty}\left(S_{\mu}\right)$ and $h$ is integrable with respect to $|d z| /|z|$ on the boundary of $S_{\mu}, \partial S_{\mu}$, then $h(T)$ is bounded since it can be represented by a contour integral which converges absolutely in the operator norm. In order to show that the conditions we derive guarantee a bounded functional calculus, we need the Convergence Lemma for approximate operators $[13,2]$.

LEMma 2.2. (Convergence Lemma) Suppose $T$ is a one-to-one operator of type $\omega$ with dense domain and dense range in $\mathcal{X}$, and that $\mu>\omega$. Let $\left\{f_{\alpha}\right\}$ be a uniformly bounded net of functions in $H^{\infty}\left(S_{\mu}\right)$ which converges to a function $f$ in $H^{\infty}\left(S_{\mu}\right)$, uniformly on compact subsets of $S_{\mu}$. Suppose further that the operators $f_{\alpha}(T)$ are uniformly bounded on $\mathcal{X}$. Then $f_{\alpha}(T) u$ converges to $f(T) u$ for all $u$ in $\mathcal{X}$, and consequently $f(T)$ is a bounded linear operator on $\mathcal{X}$, and $\|f(T)\| \leqslant \sup \left\|f_{\alpha}(T)\right\|$. 


\section{Function Theory on a Sector}

In this section we develop the relevant function theory. Fix $\pi>\nu>\mu \geqslant 0$. We wish to take a dyadic decomposition of the boundary of a sector between $S_{\nu}$ and $S_{\mu}$ in such a way that the length of each dyadic piece is less than the distance from its centre to the boundary of both $S_{\nu}$ and $S_{\mu}$. Accordingly let $\beta=(\nu+\mu) / 2$, and choose $\rho>1$ so that

$$
\rho-1<\frac{1}{2} \operatorname{dist}\left(e^{i \beta}, S_{\mu}\right)=\frac{1}{2} \sin \left(\frac{\nu-\mu}{2}\right) .
$$

Define a dyadic decomposition of the boundary $\partial S_{\beta}$ of $S_{\beta}$ by setting for $k \in \mathbb{Z}$,

$$
I_{1, k}=\left\{r e^{i \beta}: \rho^{k} \leqslant r \leqslant \rho^{k+1}\right\}, \text { and } I_{2, k}=\left\{r e^{-i \beta}: \rho^{k} \leqslant r \leqslant \rho^{k+1}\right\} .
$$

We refer to the centres of the dyadic pieces as

$$
z_{1, k}=\frac{1}{2}\left(\rho^{k}+\rho^{k+1}\right) e^{i \beta}, \text { and } z_{2, k}=\frac{1}{2}\left(\rho^{k}+\rho^{k+1}\right) e^{-i \beta} .
$$

For $m=1,2$, let $\left\{e_{m, 0, j}\right\}_{j=0}^{\infty}$ be an orthonormal basis of polynomials of $L^{2}\left(I_{m, 0}\right.$, $|d z| /|z|)$ obtained from the orthonormalisation of the sequence $\left\{z^{j}\right\}_{j=0}^{\infty}$. Thus each $e_{m, 0, j}$ is a polynomial of degree $j$ which satisfies

$$
\int_{I_{m, 0}} w^{\ell} e_{m, 0, j}(w) \frac{|d w|}{|w|}=0 \text { for all } \ell<j .
$$

For $z \in I_{m, k}$ and $k \in \mathbb{Z}$ set

$$
e_{m, k, j}(z)= \begin{cases}e_{m, 0, j}\left(z \rho^{-k}\right) & z \in I_{m, k} \\ 0 & \text { otherwise }\end{cases}
$$

Note that $\left\{e_{m, k, j}\right\}_{j=0}^{\infty}$ is an orthonormal basis of polynomials of $L^{2}\left(I_{m, k},|d z| /|z|\right)$, and that together the $e_{m, k, j}$ 's form an orthonormal basis for $L^{2}\left(\partial S_{\beta},|d z| /|z|\right)$.

For $z$ on the boundary of $S_{\beta}$ and $\zeta$ in its interior, define the kernel $K$ by

$$
K(z, \zeta)=\frac{z^{1 / 2} \zeta^{1 / 2}}{(z-\zeta)}
$$

and observe from Cauchy's theorem that, for $h \in H^{\infty}\left(S_{\beta}\right)$,

$$
h(\zeta)=\frac{1}{2 \pi i} \int_{\partial S_{\beta}} h(z) K(z, \zeta) \frac{d z}{z} .
$$

For each $e_{m, k, j}$ define the holomorphic function $\Phi_{m, k, j}$ on $S_{\beta}$ by

$$
\Phi_{m, k, j}(\zeta)=\frac{1}{2 \pi i} \int_{\partial S_{\beta}} e_{m, k, j}(z) K(z, \zeta) \frac{d z}{z} .
$$


Straightforward calculations show that for $\zeta \in S_{\mu}$, then $\left|\Phi_{m, k, j}(\zeta)\right| \leqslant c(\nu-\mu)^{-1}$. It is also interesting to note that $\Phi_{m, k, j}(\zeta)=\Phi_{m, 0, j}\left(\zeta \rho^{-k}\right)$.

The following proposition shows how functions in $H^{\infty}\left(S_{\beta}\right)$ can be decomposed into sums of the $\Phi_{m, k, j}$ 's. We note however that, although we provide a method for achieving such a decomposition, it is not unique.

Proposition 3.1. If $h \in H^{\infty}\left(S_{\beta}\right)$, then, as an element of $H^{\infty}\left(S_{\mu}\right)$,

$$
h(\zeta)=\sum_{m, k, j} \alpha_{m, k, j} \Phi_{m, k, j}(\zeta)
$$

where

$$
\alpha_{m, k, j}=\int_{I_{m, k}} h(z) \overline{e_{m, k, j}(z)} \frac{|d z|}{|z|}
$$

satisfies

$$
\left|\alpha_{m, k, j}\right| \leqslant \sqrt{\log (\rho)}\|h\|_{\infty} .
$$

Proof: First note that

$$
\sup _{m, k, j}\left|\alpha_{m, k, j}\right|^{2} \leqslant \sup _{m, k} \sum_{j}\left|\alpha_{m, k, j}\right|^{2}=\sup _{m, k} \int_{I_{m, k}}|h(z)|^{2} \frac{|d z|}{|z|} \leqslant \log (\rho)\|h\|_{\infty}^{2} .
$$

Further, for fixed $\zeta \in S_{\mu}, K \in L^{1}\left(\partial S_{\beta},|d z| /|z|\right)$, hence (3) and (4) give

$$
\begin{aligned}
h(\zeta) & =\lim _{n \rightarrow \infty} \sum_{k=-n}^{n} \sum_{m=1}^{2} \int_{I_{m, k}} h(z) K(z, \zeta) \frac{d z}{z} \\
& =\lim _{n \rightarrow \infty} \sum_{k=-n}^{n} \sum_{m=1}^{2} \int_{I_{m, k}} \sum_{j} \alpha_{m, k, j} e_{m, k, j}(z) K(z, \zeta) \frac{d z}{z} \\
& =\lim _{n \rightarrow \infty} \sum_{k=-n}^{n} \sum_{m, j} \alpha_{m, k, j} \Phi_{m, k, j}(\zeta) .
\end{aligned}
$$

Thus the proposition will follow if the sequence $\left\{\Phi_{m, k, j}(\zeta)\right\}$ has sufficient decay, a fact proven in the following lemma.

Lemma 3.2. If $\zeta \in S_{\mu}$ and $n$ is chosen so that $\rho^{n} \leqslant|\zeta| \leqslant \rho^{n+1}$, then

$$
\left|\Phi_{m, k, j}(\zeta)\right| \leqslant c \rho^{-|k-n| / 2} 2^{-j},
$$

so that for $p>0$

$$
\sup _{\zeta \in S_{\mu}} \sum_{m, k, j}\left|\Phi_{m, k, j}(\zeta)\right|^{p} \leqslant c
$$

where $c$ depends only on $p$. 
Proof: Fix $\zeta \in S_{\mu}$ and choose $n$ so that $\rho^{n} \leqslant|\zeta| \leqslant \rho^{n+1}$. Let $B_{m, k}$ denote the ball of radius $\left(\rho^{k+1}-\rho^{k}\right)$ centred at $z_{m, k}$. Observe that $B_{m, k}$ does not intersect $S_{\mu}$ or $\partial S_{\nu}$. Using the first observation and (2) one sees that

$$
\sup _{z \in B_{m, k}}|K(z, \zeta)| \leqslant c \rho^{-\frac{1}{2}|k-n|}
$$

Let

$$
\sum_{j=0}^{\infty} b_{m, k, j}\left(\frac{z-z_{m, k}}{\rho^{k+1}-\rho^{k}}\right)^{j}
$$

be the normalised power series expansion of $K(z, \zeta)$ on $B_{m, k}$, so that

$$
\left\{\sum_{j=0}^{\infty}\left|b_{m, k, j}\right|^{2}\right\}^{1 / 2}=\left\{\int_{\partial B_{m, k}}|K(z, \zeta)|^{2} \frac{|d z|}{2 \pi\left(\rho^{k+1}-\rho^{k}\right)}\right\}^{1 / 2} \leqslant c \rho^{-|k-n| / 2} .
$$

Note that $I_{m, k}$ is contained in a ball of half the radius of $B_{m, k}$. Thus for $z \in I_{m, k}$ and $N \in \mathbb{Z}$

$$
\sum_{j=N}^{\infty}\left|b_{m, k, j}\left(\frac{z-z_{m, k}}{\rho^{k+1}-\rho^{k}}\right)^{j}\right| \leqslant c \rho^{-|k-n| / 2} 2^{-N} .
$$

Finally, from (1), $e_{m, k, j}$ is orthogonal to $\left(z-z_{m, k}\right)^{s}$ for $s<j$, thus

$$
\begin{aligned}
\left|\Phi_{m, k, j}(\zeta)\right| & =\left|\frac{1}{2 \pi i} \int_{\partial S_{\beta}} e_{m, k, j}(z) K(z, \zeta) \frac{d z}{z}\right| \\
& =\left|\frac{1}{2 \pi i} \int_{I_{m, k}} \sum_{s=j}^{\infty} e_{m, k, j}(z) b_{m, k, s}\left(\frac{z-z_{m, k}}{\rho^{k+1}-\rho^{k}}\right)^{s} \frac{d z}{z}\right| \\
& \leqslant \frac{1}{2 \pi} \int_{I_{m, k}}\left|e_{m, k, j}(z)\right| \sum_{s=j}^{\infty}\left|b_{m, k, s}\left(\frac{z-z_{m, k}}{\rho^{k+1}-\rho^{k}}\right)^{s}\right| \frac{|d z|}{|z|} \\
& \leqslant c \rho^{-1 / 2|k-n|_{2}-j} .
\end{aligned}
$$

We require two more sequences obtained from the $\Phi_{m, k, j}$ 's. Let

$$
\Lambda_{m, k, j}(\zeta) \Psi_{m, k, j}^{2}(\zeta)=\Phi_{m, k, j}(\zeta)
$$

be the inner-outer factorisation of $\Phi_{m, k, j}$ on $S_{\mu}$, with $\Lambda_{m, k, j}$ the inner factor and $\Psi_{m, k, j}^{2}$ the outer factor. Set

$$
\widetilde{\Psi}_{m, k, j}(\zeta)=\Lambda_{m, k, j}(\zeta) \Psi_{m, k, j}(\zeta)
$$

This gives a factorisation $\Phi_{m, k, j}=\Psi_{m, k, j} \tilde{\Psi}_{m, k, j}$ with the property that, for $\zeta \in \partial S_{\mu}$,

$$
\left|\Psi_{m, k, j}(\zeta)\right|=\left|\tilde{\Psi}_{m, k, j}(\zeta)\right|=\left|\Phi_{m, k, j}(\zeta)\right|^{1 / 2}
$$


and hence, for $p>0$,

$$
\sup _{\zeta \in S_{\mu}} \sum_{m, k, j}\left|\Phi_{m, k, j}(\zeta)\right|^{p} \leqslant c
$$

We remark that any other factorisation with this property would suffice.

\section{Operator Theory}

Let $A$ be an operator of type $\omega$ acting on a complex Banach space $\mathcal{X}$. Let $\Phi_{m, k, j}$ be defined as in Section 3. We present the equivalence of $A$ having a bounded functional calculus with $A$ satisfying discrete weak quadratic estimates.

We say that $A$ has a bounded $H^{\infty}\left(S_{\theta}\right)$ functional calculus if $h(A) \in \mathcal{L}(\mathcal{X})$ for all $h \in H^{\infty}\left(S_{\theta}\right)$ with

$$
\|h(L)\| \leqslant c\|h\|_{\infty}
$$

We say that $A$ satisfies discrete weak quadratic estimates $(\mathcal{W})$ if

$$
\sum_{m, k, j}\left|\left\langle\Phi_{m, k, j}(A) u, v\right\rangle\right| \leqslant c\|u\|\|v\|, \quad u \in \mathcal{X}, v \in \mathcal{X}^{*} .
$$

By analogy with Theorems 4.2 and 4.4 of [6] one has the following theorem.

THEOREM 4.1. Let $A$ be an operator of type $\omega$ acting on a complex Banach space $\mathcal{X}$ which is one-to-one and has dense domain and dense range. If $A$ satisfies $(\mathcal{W})$ then $A$ satisfies $\left(\mathcal{F}_{\beta}\right)$, and conversely if $A$ satisfies $\left(\mathcal{F}_{\mu}\right)$ then $A$ satisfies $(\mathcal{W})$.

Proof: Let $h \in H^{\infty}\left(S_{\beta}\right)$ and decompose $h$ as in Proposition 3.1,

$$
h(\zeta)=\sum_{m, k, j} \alpha_{m, k, j} \Phi_{m, k, j}(\zeta)
$$

where the $\alpha_{m, k, j}$ 's are as in (5). Observe that if $(\mathcal{W})$ holds then using $(6)$ one sees that for all $u \in \mathcal{X}$, and $v \in \mathcal{X}^{*}$,

$$
|\langle h(A) u, v\rangle|=\left|\sum_{m, k, j}\left\langle\alpha_{m, k, j} \Phi_{m, k, j}(A) u, v\right\rangle\right| \leqslant c\|h\|_{\infty}\|u\|\|v\|,
$$

hence $A$ satisfies $\left(\mathcal{F}_{\beta}\right)$.

Conversely, suppose $A$ satisfies $\left(\mathcal{F}_{\mu}\right)$. For $u, v$ fixed, choose unimodular constants $b_{m, k, j}^{u, v}$ so that

$$
\sum_{m, k, j}\left|\left\langle\Phi_{m, k, j}(A) u, v\right\rangle\right|=\left\langle\sum_{m, k, j} b_{m, k, j}^{u, v} \Phi_{m, k, j}(A) u, v\right\rangle .
$$


Now the hypothesis, Lemma 3.2, and the Convergence Lemma show that the above is bounded by

$$
c \sup _{\zeta \in S_{\mu}}\left|\sum_{m, k, j} b_{m, k, j}^{u, v} \Phi_{m, k, j}(\zeta)\right|\|u\|\|v\| \leqslant c\|u\|\|v\| .
$$

Thus $(\mathcal{W})$ holds.

We now show the equivalence of discrete weak quadratic estimates with discrete quadratic estimates when $\mathcal{X}=L^{p}$.

Let $T$ be an operator of type $\omega$ acting on $L^{p}(\Omega), 1<p<\infty$, where $(\Omega, d x)$ is a $\sigma$-finite measure space. Let $q$ be the conjugate exponent to $p$ and let $T^{*}$ be the adjoint of $T$ with respect to the bilinear pairing between $L^{p}(\Omega)$ and $L^{q}(\Omega)$. Let $\Psi_{m, k, j}$ and $\widetilde{\Psi}_{m, k, j}$ be defined as in Section 3. Let $f \in L^{p}(\Omega)$ and $g \in L^{q}(\Omega)$. Consider the following discrete quadratic estimates which $T$ might satisfy.

$$
\begin{gathered}
\left\|\left\{\sum_{m, k, j}\left|\Psi_{m, k, j}(T) f(\cdot)\right|^{2}\right\}^{1 / 2}\right\|_{p} \leqslant c\|f\|_{p}, \\
\left\|\left\{\sum_{m, k, j}\left|\tilde{\Psi}_{m, k, j}\left(T^{*}\right) g(\cdot)\right|^{2}\right\}^{1 / 2}\right\|_{q} \leqslant c\|g\|_{q}, \\
\left\|\left\{\sum_{m, k, j}\left|2^{-j / 2} \Psi_{m, k, j}(T) f(\cdot)\right|^{2}\right\}^{1 / 2}\right\|_{p} \leqslant c\|f\|_{p}, \\
\left\|\left\{\sum_{m, k, j}\left|2^{-j / 2} \tilde{\Psi}_{m, k, j}\left(T^{*}\right) g(\cdot)\right|^{2}\right\}^{1 / 2}\right\|_{q} \leqslant c\|g\|_{q} .
\end{gathered}
$$

Clearly $(\mathcal{S})$ and $\left(\mathcal{S}^{*}\right)$ imply $(\mathbb{S})$ and $\left(\mathbb{S}^{*}\right)$ respectively. Moreover if $\mathcal{X}=L^{p}(\Omega)$ and $(\mathcal{S})$ and $\left(\mathcal{S}^{*}\right)$ hold, then $(\mathcal{W})$ holds for $A=T$.

THEOREM 4.2. Let $T$ be an operator of type $\omega$ acting on $L^{p}(\Omega), 1<p<\infty$, which is one-to-one and has dense domain and dense range, then;

(i) If $T$ satisfies $\left(\mathcal{F}_{\mu}\right)$ then $(\mathcal{S})$ and $\left(\mathcal{S}^{*}\right)$ hold.

(ii) If $(\mathcal{S})$ and $\left(\mathcal{S}^{*}\right)$ hold then $T$ satisfies $\left(\mathcal{F}_{\beta}\right)$.

(iii) If $(\mathbb{S})$ and $\left(\mathbb{S}^{*}\right)$ hold then $T$ satisfies $\left(\mathcal{F}_{\nu}\right)$.

Proof: To see (i), apply the randomisation lemma as in [6] (or in the proof of Theorem 4.3 ) to the sum in $(\mathcal{S})$ to obtain

$$
\left\|\left\{\sum_{m, k, j}\left|\Psi_{m, k, j}(T) f(\cdot)\right|^{2}\right\}^{1 / 2}\right\|_{p} \leqslant c \sup _{\|a\|_{\infty}=1}\left\|\left(\sum_{m, k, j} a_{m, k, j} \Psi_{m, k, j}(T)\right) f\right\|_{p} .
$$

Therefore $(\mathcal{S})$ follows from the hypothesis and $(7)$. 
(ii) Let $h \in H^{\infty}\left(S_{\beta}\right)$ and again decompose $h$ as in Proposition 3.1. Hence

$$
\begin{aligned}
\langle h(T) f, g\rangle & =\sum_{m, k, j}\left\langle\alpha_{m, k, j} \Phi_{m, k, j}(T) f, g\right\rangle \\
& =\sum_{m, k, j}\left\langle\alpha_{m, k, j} \Psi_{m, k, j}(T) f, \tilde{\Psi}_{m, k, j}\left(T^{*}\right) g\right\rangle \\
& =\int_{\Omega} \sum_{m, k, j} \alpha_{m, k, j} \Psi_{m, k, j}(T) f(x) \tilde{\Psi}_{m, k, j}\left(T^{*}\right) g(x) d x
\end{aligned}
$$

and so

$$
|\langle h(T) f, g\rangle| \leqslant\|h\|_{\infty}\left\|\left\{\sum_{m, k, j}\left|\Psi_{m, k, j}(T) f(\cdot)\right|^{2}\right\}^{1 / 2}\right\|_{p}\left\|\left\{\sum_{m, k, j}\left|\widetilde{\Psi}_{m, k, j} g(\cdot)\right|^{2}\right\}^{1 / 2}\right\|_{q} .
$$

Thus $T$ satisfies $\left(\mathcal{F}_{\beta}\right)$ since $(\mathcal{S})$ and $\left(\mathcal{S}^{*}\right)$ show that the last line of the above is bounded by $c\|h\|_{\infty}\|f\|_{p}\|g\|_{q}$.

To see (iii) note that if $h \in H^{\infty}\left(S_{\nu}\right)$ then by considering the power series of $h$ on $B_{m, k}$ as defined in the proof of Lemma 3.2 and arguing similarly one can show that

$$
\alpha_{m, k, j}=\int_{I_{m, k}} h(z) \overline{e_{m, k, j}(z)} \frac{|d z|}{|z|} \leqslant c\|h\|_{\infty} 2^{-j} .
$$

A calculation similar to the one used to prove $(i i)$ shows that $T$ satisfies $\left(\mathcal{F}_{\nu}\right)$.

We now treat the case of several commuting operators on $L^{p}(\Omega), 1<p<\infty$. Let $T=\left(T_{1}, T_{2}, \ldots, T_{d}\right)$ be a $d$-tuple of commuting operators with $T_{i}$ of type $\omega_{i}$ and possessing a bounded $H^{\infty}\left(S_{\mu_{i}}\right)$ functional calculus for some $\mu_{i}>\omega_{i}$. Choose $\beta_{i}>\mu_{i}$, and set $(\beta)=\left(\beta_{1}, \beta_{2}, \ldots, \beta_{d}\right), S_{(\beta)}=S_{\beta_{1}} \times S_{\beta_{2}} \times \ldots S_{\beta_{d}}$, and let $H^{\infty}\left(S_{(\beta)}\right)$ denote the space of bounded holomorphic functions on $S_{(\beta)}$. In [1] Albrecht proved with a careful application of the Rademacher inequalities that $T$ has a bounded $H^{\infty}\left(S_{(\beta)}\right)$ functional calculus, that is for $h(z)=h\left(z_{1}, z_{2}, \ldots, z_{d}\right) \in H^{\infty}\left(S_{(\beta)}\right)$

$$
\|h(T)\|=\left\|h\left(T_{1}, T_{2}, \ldots, T_{d}\right)\right\| \leqslant c\|h\|_{\infty} .
$$

See also [3]. We present an alternative proof of Albrecht's theorem using discrete quadratic estimates.

Let $(m, k, j)$ be a multi-index $\left(m_{1}, \ldots, m_{d}, k_{1}, \ldots, k_{d}, j_{1}, \ldots, j_{d}\right)$ with $m_{i}=1,2, k_{i} \in$ $\mathbb{Z}$, and $j_{i}=0,1,2, \ldots$. Construct functions $e_{m_{1}, k_{1}, j_{1}}, \Phi_{m_{1}, k_{1}, j_{1}}, \Psi_{m_{1}, k_{1}, j_{1}}$, and $\widetilde{\Psi}_{m_{1}, k_{1}, j_{1}}$ as in Section 3. For $\zeta$ in $S_{(\beta)}$ set

$$
\Phi_{(m, k, j)}(\zeta)=\Phi_{m_{1}, k_{1}, j_{1}}\left(\zeta_{1}\right) \Phi_{m_{2}, k_{2}, j_{2}}\left(\zeta_{2}\right) \ldots \Phi_{m_{d}, k_{d}, j_{d}}\left(\zeta_{d}\right) .
$$

$\widetilde{\Psi}_{(m, k, j)}, \Psi_{(m, k, j)}$ and $e_{(m, k, j)}$ are defined similarly. Let

$$
J_{(m, k)}=I_{m_{1}, k_{1}} \times I_{m_{2}, k_{2}} \times \cdots \times I_{m_{d}, k_{d}} .
$$


Every element $h \in H^{\infty}\left(S_{(\beta)}\right)$ has a decomposition as a sum of the $\Phi_{(m, k, j)}$ 's. In fact for $\zeta \in S_{\mu}$ one has,

$$
h(\zeta)=\sum_{(m, k, j)} \alpha_{(m, k, j)} \Phi_{(m, k, j)}(\zeta)
$$

where

$$
\alpha_{(m, k, j)}=\int_{J_{(m, k)}} h(z) \overline{e_{(m, k, j)}(z)} \frac{\left|d z_{1}\right|}{\left|z_{1}\right|} \frac{\left|d z_{2}\right|}{\left|z_{2}\right|} \ldots \frac{\left|d z_{d}\right|}{\left|z_{d}\right|} .
$$

As before,

$$
\begin{aligned}
\sup _{(m, k, j)}\left|\alpha_{(m, k, j)}\right|^{2} \leqslant \sup _{(m, k)} \sum_{(j)}\left|\alpha_{(m, k, j)}\right|^{2} & =\sup _{(m, k)} \int_{J_{(m, k)}}|h(z)|^{2} \frac{\left|d z_{1}\right|}{\left|z_{1}\right|} \frac{\left|d z_{2}\right|}{\left|z_{2}\right|} \ldots \frac{\left|d z_{d}\right|}{\left|z_{d}\right|} \\
& \leqslant c\|h\|_{\infty}^{2} .
\end{aligned}
$$

With everything defined as above we have the following theorem.

THEOREM 4.3. (Albrecht) For $1 \leqslant i \leqslant d$ fix $0 \leqslant \omega_{i}<\mu_{i}<\beta_{i}<\pi$. If $T=$ $\left(T_{1}, T_{2}, \ldots, T_{d}\right)$ is a d-tuple of commuting operators, with $T_{i}$ of type $\omega_{i}$ and possessing a bounded $H^{\infty}\left(S_{\mu_{i}}\right)$ functional calculus, then $T$ has a bounded $H^{\infty}\left(S_{(\beta)}\right)$ functional calculus.

Proof: As in the proof of Theorem 4.1

$$
|\langle h(T) f, g\rangle| \leqslant\left\|\left\{\sum_{(m, k, j)}\left|\Psi_{(m, k, j)}(T) f(\cdot)\right|^{2}\right\}^{1 / 2}\right\|\left\|\left\{\sum_{(m, k, j)}\left|\tilde{\Psi}_{(m, k, j)}\left(T^{*}\right) g(\cdot)\right|^{2}\right\}^{1 / 2}\right\|_{q} .
$$

Define the product of Rademacher functions on $[0,1]^{(3 d)}$ by setting

$$
r_{(m, k, j)}(t)=r_{m_{1}}\left(t_{1}\right) \ldots r_{m_{d}}\left(t_{d}\right) r_{k_{1}}\left(t_{d+1}\right) \ldots r_{k_{d}}\left(t_{2 d}\right) r_{j_{1}}\left(t_{2 d+1}\right) \ldots r_{j_{d}}\left(t_{3 d}\right)
$$

where each of the $r$ 's is the Rademacher function corresponding to its subscript. Letting $d t=d t_{1} d t_{2} \ldots d t_{3 d}$ one has, using the inequalities for Rademacher functions [16], that

$$
\begin{aligned}
\left\|\left\{\sum_{(m, k, j)}\left|\Psi_{(m, k, j)}(T) f(\cdot)\right|^{2}\right\}^{1 / 2}\right\|_{p}^{p} \\
\quad=\int_{\Omega}\left(\int_{[0,1]^{(3 d)}}\left|\sum_{(m, k, j)} r_{(m, k, j)}(t) \Psi_{(m, k, j)}(T) f(x)\right|^{2} d t\right)^{p / 2} d x \\
\quad \leqslant c \int_{[0,1]^{(3 d)}} \int_{\Omega}\left|\sum_{(m, k, j)} r_{(m, k, j)}(t) \Psi_{(m, k, j)}(T) f(x)\right|^{p} d x d t .
\end{aligned}
$$


For $i=1,2, \ldots, d$ and $t \in[0,1]^{(3 d)}$, set

$$
F_{i}(t, \zeta)=\sum_{m, k, j} r_{m_{i}}\left(t_{i}\right) r_{k_{i}}\left(t_{d+i}\right) r_{j_{i}}\left(t_{2 d+i}\right) \Psi_{m_{i}, k_{i}, j_{i}}(\zeta)
$$

Now $T_{i}$ has a bounded $H^{\infty}\left(S_{\mu_{i}}\right)$ functional calculus hence, by Lemma 3.2,

$$
\sup _{t}\left\|F_{i}(t, T)\right\| \leqslant \sup _{t} \sup _{\zeta \in S_{\left(\mu_{i}\right)}}\left|\sum_{m, k, j} r_{m_{i}}\left(t_{i}\right) r_{k_{i}}\left(t_{d+i}\right) r_{j_{i}}\left(t_{2 d+i}\right) \Psi_{m_{i}, k_{i}, j_{i}}(\zeta)\right| \leqslant c .
$$

It follows that ( 8 ) is bounded by

$$
\sup _{t}\left\|\left(F_{1}\left(t, T_{1}\right)\left(F_{2}\left(t, T_{2}\right)\left(\ldots\left(F_{d}\left(t, T_{d}\right) f\right)\right)\right)\right)\right\|_{p}^{p} \leqslant c\|f\|_{p}^{p} .
$$

Using this, together with an analogous calculation with $T^{*}$, we have

$$
|\langle h(T) f, g\rangle| \leqslant c\|f\|_{p}\|g\|_{q}
$$

so that $T$ has a bounded $H^{\infty}\left(S_{(\beta)}\right)$ functional calculus.

REMARK. Subsequent to the first draft of the current work, Lancien, Lancien and Le Merdy have shown how to use our discrete weak quadratic estimates to prove the boundedness of joint functional calculi in various classes of Banach spaces $[10,11]$.

\section{Several Points of Contact.}

In Sections 3 and 4 we developed some necessary and sufficient conditions for an operator to have a bounded $H^{\infty}$ functional calculus on an open set whose boundary touches its spectrum nontangentially at the two points 0 and $\infty$. In this section we generalise these arguments to the case of several points of contact. We begin as before with the function theory.

Let $\Delta$ be a compact subset of $\mathbb{C}$. We consider three bounded open sets, $\mathcal{O}_{1} \subset \mathcal{O}_{2} \subset$ $\mathcal{O}_{3}$, with connected closures which contain $\Delta$, and whose piecewise smooth boundaries touch $\Delta$ nontangentially at finitely many points $Z=\left\{z_{1}, z_{2}, \ldots, z_{N}\right\}$. We further require that the boundaries are piecewise linear in a neighbourhood $Z$, meet each other nontangentially at $Z$, and that $\mathcal{O}_{2}$ is the interior of its closure. The nontangentiality gives that for $w_{n} \in \partial \mathcal{O}_{n}, n=1,2,3$, and $n \neq j$,

$$
\operatorname{dist}\left(w_{n}, \Delta\right) \approx \inf _{1 \leqslant i \leqslant N} \operatorname{dist}\left(w_{n}, z_{i}\right) \text {, and } \operatorname{dist}\left(w_{n}, \partial \mathcal{O}_{j}\right) \approx \inf _{1 \leqslant i \leqslant N} \operatorname{dist}\left(w_{n}, z_{i}\right) .
$$

Construct a reproducing kernel analogous to $K$ for $\mathcal{O}_{2}$ by choosing for $1 \leqslant i \leqslant N$ a biholomorphic mapping $\varphi_{i}$ of a neighbourhood of the closure of $\mathcal{O}_{3}$ which maps $\mathcal{O}_{3}$ to a bounded domain contained in the right half plane and takes $z_{i}$ to 0 . Set

$$
\mathcal{K}(z, \zeta)=\frac{\left(\prod_{i=1}^{N} \varphi_{i}(\zeta) \varphi_{i}(z)\right)^{1 / 2}}{(z-\zeta)}, \quad \text { and } \quad d \lambda=\frac{d z}{\prod_{i=1}^{N} \varphi_{i}(z)}
$$


The differentiability of the $\varphi_{i}$ 's show that for $\zeta \in \mathcal{O}_{1}$,

$$
\int_{\partial \mathcal{O}_{2}}|\mathcal{K}(z, \zeta)||d \lambda| \leqslant c \int_{\partial \mathrm{O}_{2}} \frac{\left|\prod_{i=1}^{N}\left(z_{i}-\zeta\right)\left(z_{i}-z\right)\right|^{1 / 2}}{|z-\zeta| \prod_{i=1}^{N}\left|z_{i}-z\right|}|d z| \leqslant c .
$$

Therefore $\mathcal{K}(\cdot, \zeta)$ is bounded in $L^{1}\left(\partial \mathcal{O}_{2},|d \lambda|\right)$ uniformly for $\zeta \in \mathcal{O}_{1}$. Let $h \in H^{\infty}\left(\mathcal{O}_{2}\right)$, and observe from Cauchy's theorem that for $\zeta \in \mathcal{O}_{2}$,

$$
h(\zeta)=\frac{1}{2 \pi i} \int_{\partial \mathcal{O}_{2}} h(z) \mathcal{K}(z, \zeta) d \lambda .
$$

We now construct a decomposition of $\partial \mathrm{O}_{2}$. Let $\Gamma_{0}$ be the union of all the closed curves in $\partial \mathcal{O}_{2}$ which do not intersect $Z$. Choose subarcs $\left\{\gamma_{0, k}\right\}_{k=0}^{k_{0}}$ of $\Gamma_{0}$ so that

$$
\delta_{0, k}=\sup \left\{|y-\tilde{y}|: y, \tilde{y} \in \gamma_{0, k}\right\} \leqslant \frac{1}{3} \operatorname{dist}\left(\gamma_{0, k}, \partial \mathcal{O}_{1} \cup \partial \mathcal{O}_{3}\right) .
$$

Decompose the remainder of the boundary into curves $\Gamma_{m}, 1 \leqslant m \leqslant N_{1}$ which intersect $Z$ at one of their end points. Let $y_{m, 0}$ and $y_{m, \infty}$ denote the end points of $\Gamma_{m}$ with $y_{m, \infty} \in Z$. Choose points $y_{m, 1}, y_{m, 2}, \ldots$ along $\Gamma_{m}$ so that the subarcs $\left\{\gamma_{m, k}\right\}_{k=0}^{\infty}$ which connect $y_{m, k}$ to $y_{m, k+1}$ partition $\Gamma_{m}$ and satisfy

$$
\delta_{m, k}=\sup \left\{|y-\widetilde{y}|: y, \widetilde{y} \in \gamma_{m, k}\right\}=\frac{1}{3} \operatorname{dist}\left(\gamma_{m, k}, \partial \mathcal{O}_{1} \cup \partial \mathcal{O}_{3}\right) .
$$

Since the boundaries of the $\mathcal{O}_{i}$ 's are piecewise linear in a neighbourhood of the $z_{i}$ 's and meet each other nontangentially at the $z_{i}$ 's, it follows that $\delta_{m, k}$ eventually tends exponentially to 0 as $k \longrightarrow \infty$. Equivalently, $\left\{y_{m, k}\right\}$ eventually tends exponentially to $y_{m, \infty}$. Hence there exists a positive integer $k_{m}$ and a constant $\rho_{m}>1$ such that, for $k>k_{m}$

$$
\left|y_{m, k}-y_{m, \infty}\right|=c \rho_{m}^{-\left(k-k_{m}\right)} .
$$

If $k \leqslant k_{m}$, then the fact that the $\varphi_{i}$ 's are biholomorphic shows that

$$
\begin{aligned}
\sup _{z \in \gamma_{m, k}}|\mathcal{K}(z, \zeta)| & \approx\left|\prod_{i=1}^{N} \varphi_{i}(\zeta) \varphi_{i}(z)\right|^{1 / 2} \\
& \approx\left|\prod_{i=1}^{N}\left(z_{i}-\zeta\right)\left(z_{i}-z\right)\right|^{1 / 2} \\
& \approx \inf _{(1 \leqslant i \leqslant N)}\left|\zeta-z_{i}\right|^{1 / 2}
\end{aligned}
$$

Let $\zeta \in \mathcal{O}_{1}$, and choose $r_{m}$ so that

$$
\rho_{m}^{-\left(r_{m}-k_{m}\right)} \leqslant\left|\zeta-y_{m, \infty}\right| \leqslant \rho_{m}^{-\left(\left(r_{m}-1\right)-k_{m}\right)} .
$$


From (10) and (12) one has, for $k>k_{m}$ and $z \in \gamma_{m, k}$,

$$
\begin{aligned}
|\mathcal{K}(z, \zeta)| & \approx \frac{\left|\prod_{i=1}^{N}\left(z_{i}-\zeta\right)\left(z_{i}-z\right)\right|^{1 / 2}}{\left|z-y_{m, \infty}\right|+\left|\zeta-y_{m, \infty}\right|} \\
& \approx \frac{\left(\rho_{m}^{-\left(k-k_{m}\right)} \rho_{m}^{-\left(r_{m}-k_{m}\right)}\right)^{1 / 2}}{\rho_{m}^{-\left(k-k_{m}\right)}+\rho_{m}^{-\left(r_{m}-k_{m}\right)}} \inf _{\left(z_{i} \neq y_{m, \infty}\right)}\left|\zeta-z_{i}\right|^{1 / 2} \\
& \approx \rho_{m}^{\left|k-r_{m}\right| / 2} \inf _{\left(z_{i} \neq y_{m, \infty}\right)}\left|\zeta-z_{i}\right|^{1 / 2} .
\end{aligned}
$$

Let $\left\{e_{m, k, j}\right\}_{j=0}^{\infty}$ be an orthonormal basis of polynomials of $L^{2}\left(\gamma_{m, k},|d \lambda|\right)$ obtained from the orthonormalisation of the sequence $\left\{z^{j}\right\}_{j=0}^{\infty}$. As before, each $e_{m, k, j}$ is a polynomial of degree $j$ which satisfies

$$
\int_{\gamma_{m, k}} z^{\ell} e_{m, k, j}(z)|d \lambda|=0 \quad \text { for all } \ell<j .
$$

For each $e_{m, k, j}$ define the holomorphic function $\Phi_{m, k, j}$ on $\mathcal{O}_{2}$ by the formula

$$
\Phi_{m, k, j}(\zeta)=\frac{1}{2 \pi i} \int_{\partial \mathcal{O}_{2}} e_{m, k, j}(z) \mathcal{K}(z, \zeta) d \lambda
$$

For $\zeta \in \mathcal{O}_{1}$ and $r_{m}$ as in (12), the estimates in (11) and (13) show, using the same methods used in Lemma 3.2 , that

$$
\left|\Phi_{m, k, j}(\zeta)\right| \leqslant \begin{cases}c 2^{-j} \inf _{(1 \leqslant i \leqslant N)}\left|\zeta-z_{i}\right|^{1 / 2} & \text { if } k \leqslant k_{m}, \\ c \rho_{m}^{-\left|k-r_{m}\right| / 2} 2^{-j} \inf _{\left(z_{i} \neq y_{m, \infty}\right)}\left|\zeta-z_{i}\right|^{1 / 2} & \text { if } k>k_{m} .\end{cases}
$$

Note that (14) implies that, if $h \in H^{\infty}\left(\mathcal{O}_{2}\right)$, then, as an element of $H^{\infty}\left(\mathcal{O}_{1}\right)$, we may decompose $h$ as a sum of the $\Phi_{m, k, j}$ 's. In fact, we have the following analogue of Proposition 3.1.

PROPOSITION 5.1. If $h \in H^{\infty}\left(\mathcal{O}_{2}\right)$ then as an element of $H^{\infty}\left(\mathcal{O}_{1}\right)$

$$
h(\zeta)=\sum_{m, k, j} \alpha_{m, k, j} \Phi_{m, k, j}(\zeta)
$$

where

$$
\alpha_{m, k, j}=\int_{\gamma_{m, k}} h(z) \overline{e_{m, k, j}(z)}|d \lambda|
$$

As in Section 3 we need to factor the $\Phi_{m, k, j}$ 's. However, as $\mathcal{O}_{1}$ may not be simply connected, inner-outer factorisation may be unavailable. The following lemma guarantees the necessary factorisation. 
LEMMA 5.2. For $\left\{\Phi_{m, k, j}\right\}$ as above, there exist sequences of functions $\left\{\Psi_{m, k, j}\right\}$ and $\left\{\widetilde{\Psi}_{m, k, j}\right\}$ in $H^{\infty}\left(\mathcal{O}_{1}\right)$, such that for $\zeta \in \mathcal{O}_{1}$ and $r_{m}$ as in (12),

$$
\begin{gathered}
\Psi_{m, k, j}(\zeta) \tilde{\Psi}_{m, k, j}(\zeta)=\Phi_{m, k, j}(\zeta), \text { and } \\
\left|\Psi_{m, k, j}(\zeta)\right|,\left|\widetilde{\Psi}_{m, k, j}(\zeta)\right| \leqslant \begin{cases}c 2^{-j / 2} \inf _{(1 \leqslant i \leqslant N)}\left|\zeta-z_{i}\right|^{1 / 4} & \text { if } k \leqslant k_{m}, \\
c \rho_{m}^{-\left|k-r_{m}\right| / 4} 2^{-j / 2} \inf _{\left(z_{i} \neq y_{m,-\infty}\right)}\left|\zeta-z_{i}\right|^{1 / 4} & \text { if } k>k_{m} .\end{cases}
\end{gathered}
$$

Proof: Let $\varphi_{m_{R}}$ be the previously selected mapping which takes $y_{m, \infty}$ to zero. Set

$$
\widetilde{\Psi}_{m, k, j}(\zeta)= \begin{cases}2^{-j / 2}\left(\prod_{i=1}^{N} \varphi_{i}(\zeta) \varphi_{i}\left(y_{m, k}\right)\right)^{1 / 4} & \text { if } k \leqslant k_{m} \\ 2^{-j / 2} \frac{\left(\prod_{i=1}^{N} \varphi_{i}(\zeta) \varphi_{i}\left(y_{m, k}\right)\right)^{1 / 4}}{\varphi_{m_{R}}\left(y_{m, k}\right)^{1 / 2}-\varphi_{m_{R}}(\zeta)^{1 / 2}} & \text { if } k>k_{m}\end{cases}
$$

and set

$$
\Psi_{m, k, j}(\zeta)=\left(\widetilde{\Psi}_{m, k, j}(\zeta)\right)^{-1} \Phi_{m, k, j}(\zeta)
$$

The fact that the $\varphi_{i}$ 's are biholomorphic shows that, for $k \leqslant k_{m}$,

$$
\left|\tilde{\Psi}_{m, k, j}(\zeta)\right| \approx 2^{-j / 2} \inf _{(1 \leqslant i \leqslant N)}\left|\zeta-z_{i}\right|^{1 / 4}
$$

Fix $\zeta \in \mathcal{O}_{1}$, let $r_{m}$ be as in (12). From (9) and (10) one has that, for $k>k_{m}$,

$$
\begin{aligned}
\left|\widetilde{\Psi}_{m, k, j}(\zeta)\right| & \geqslant c 2^{-j / 2} \frac{\left|\prod_{i=1}^{N}\left(z_{i}-\zeta\right)\left(z_{i}-y_{m, k}\right)\right|^{1 / 4}}{\left|y_{m, k}-y_{m, \infty}\right|^{1 / 2}+\left|\zeta-y_{m, \infty}\right|^{1 / 2}} \\
& \geqslant c 2^{-j / 2} \frac{\left(\rho_{m}^{-\left(k-k_{m}\right)} \rho_{m}^{-\left(r_{m}-k_{m}\right)}\right)^{1 / 4}}{\rho_{m}^{-\left(k-k_{m}\right) / 2}+\rho_{m}^{-\left(r_{m}-k_{m}\right) / 2}} \inf _{\left(z_{i} \neq y_{m, \infty}\right)}\left|\zeta-z_{i}\right|^{1 / 4} \\
& \geqslant c 2^{-j / 2} \rho_{m}^{-\left|k-r_{m}\right| / 2} \inf _{\left(z_{i} \neq y_{m, \infty}\right)}\left|\zeta-z_{i}\right|^{1 / 4}
\end{aligned}
$$

Further estimates show that, for $k>k_{m}$,

$$
\left|\tilde{\Psi}_{m, k, j}(\zeta)\right| \approx 2^{-j / 2} \inf _{z_{i} \neq y_{m, \infty}}\left|\zeta-z_{i}\right|^{1 / 4} \rho_{m}^{-\left|k-r_{m}\right| / 4}
$$

Thus by (15) the lemma holds.

We are now ready to consider the operator theory. Let $A$ be an operator acting on a complex Banach space $\mathcal{X}$ and $T$ be an operator acting on $L^{p}(\Omega), 1<p<\infty$, with $\sigma(A)$ and $\sigma(T)$ contained in $\Delta$. We also assume that for $z$ outside $\Delta$ and $1 \leqslant i \leqslant N$

$$
\left\|(z-A)^{-1}\right\|,\left\|(z-T)^{-1}\right\| \leqslant c(\operatorname{dist}(z, \Delta))^{-1}, \text { and }
$$




$$
z_{\boldsymbol{i}} I-A \text { and } z_{\boldsymbol{i}} I-T \text { are one-to-one closed operators }
$$

with dense domain and dense range.

As before, let $u \in \mathcal{X}, v \in \mathcal{X}^{*}, f \in L^{p}(\Omega)$, and $g \in L^{q}(\Omega)$, and consider the following conditions that $A$ and $T$ might satisfy.

$$
\begin{gathered}
\sum_{m, k, j}\left|\left\langle\Phi_{m, k, j}(A) u, v\right\rangle\right| \leqslant c\|u\|\|v\|, \\
\left\|\left\{\sum_{m, k, j}\left|\Psi_{m, k, j}(T) f(\cdot)\right|^{2}\right\}^{1 / 2}\right\|_{p} \leqslant c\|f\|_{p}, \\
\left\|\left\{\sum_{m, k, j}\left|\widetilde{\Psi}_{m, k, j}\left(T^{*}\right) g(\cdot)\right|^{2}\right\}^{1 / 2}\right\|_{q} \leqslant c\|g\|_{q}, \\
\left\|\left\{\sum_{m, k, j} 2^{-j / 2}\left|\Psi_{m, k, j}(T) f(\cdot)\right|^{2}\right\}^{1 / 2}\right\|_{p} \leqslant c\|f\|_{p}, \\
\left\|\left\{\sum_{m, k, j} 2^{-j / 2}\left|\widetilde{\Psi}_{m, k, j}\left(T^{*}\right) g(\cdot)\right|^{2}\right\}^{1 / 2}\right\|_{q} \leqslant c\|g\|_{q} .
\end{gathered}
$$

An operator $L$ has a bounded $H^{\infty}(\mathcal{O})$ functional calculus if $L$ satisfies,

$$
\|h(L)\| \leqslant c\|h\|_{\infty} \quad \text { for all } h \in H^{\infty}(\mathcal{O}) .
$$

The function theory developed in this section allows one to prove the following theorems, using virtually the same proofs as Theorem 4.1 and 4.2 .

THEOREM 5.3. Let $A$ be an operator acting on a complex Banach space $\mathcal{X}$ which satisfies (16) and (17). If $A$ satisfies $(\mathcal{W})$ then $A$ satisfies $\left(\mathcal{F}_{\mathcal{O}_{2}}\right)$, and conversely if A satisfies $\mathcal{F}_{\mathcal{O}_{1}}$ then $A$ satisfies $(\mathcal{W})$.

THEOREM 5.4. Let $T$ be an operator acting on $L^{p}(\Omega), 1<p<\infty$, which satisfies (16) and (17), then;

(i) If $T$ satisfies $\left(\mathcal{F}_{\mathcal{O}_{1}}\right)$ then $(\mathcal{S})$ and $\left(\mathcal{S}^{*}\right)$ hold.

(ii) If $(\mathcal{S})$ and $\left(\mathcal{S}^{*}\right)$ hold then $T$ satisfies $\left(\mathcal{F}_{\mathrm{O}_{2}}\right)$.

(iii) If (S) and $\left(\mathbb{S}^{*}\right)$ hold then $T$ satisfies $\left(\mathcal{F}_{\mathrm{O}_{3}}\right)$.

Also, using essentially the same proof as Theorem 4.3, one can show that several commuting operators acting on $L^{p}, 1<p<\infty$, which individually satisfy $\left(\mathcal{F}_{\mathcal{O}_{1}}\right)$ admit a joint functional calculus.

More can be said for operators on Hilbert space. In [13] the second author showed that if $T$ is an operator of type $\omega$ acting on a Hilbert space with a bounded $H^{\infty}\left(S_{\nu}\right)$ functional calculus for some $\nu>\omega$, then $T$ has a bounded $H^{\infty}\left(S_{\mu}\right)$ functional calculus for every $\mu>\omega$. An analogous fact holds in this setting. Let $\mathcal{O}$ be any open set whose closure contains $\Delta$ and whose boundary touches $\Delta$ nontangentially at $z_{1}, z_{2}, \ldots, z_{N}$. With everything defined as above we have the following theorem. 
THEOREM 5.5. Let $T$ be an operator acting on a complex Hilbert space $\mathcal{H}$ which satisfies (16) and (17). If $T$ has a bounded $H^{\infty}\left(\mathcal{O}_{1}\right)$ functional calculus, then $T$ has a bounded $H^{\infty}(\mathcal{O})$ functional calculus.

Proof: To show that $T$ has a bounded $H^{\infty}(\mathcal{O})$ functional calculus, it suffices to consider $T$ restricted to the closed separable invariant subspaces generated by a vector $\gamma$ and the functional calculus of $T$, namely, the closures of subspaces of the form $\mathcal{H}_{\gamma}=$ $\left\{h(T) \gamma: h \in H^{\infty}(\mathcal{O})\right\}$. Thus, since any separable Hilbert space is unitarily equivalent to $L^{2}(\Omega)$, we may assume without loss of generality that $\mathcal{H}=L^{2}(\Omega)$. We also assume without loss of generality that $\mathcal{O} \subset \mathcal{O}_{1}$ and has a piecewise smooth boundary. Since $T$ has a bounded $H^{\infty}\left(\mathcal{O}_{1}\right)$ functional calculus, Proposition 5.1 and the constructions preceding it allow one to decompose the identity operator $I$ as follows

$$
I=\sum_{m, k} \Phi_{m, k, 0}(T)
$$

We need a somewhat different factorisation of the $\Phi_{m, k, 0}$ 's, and accordingly, we let $\varphi_{m_{R}}$ be the previously selected mapping which takes $y_{m, \infty}$ to zero, and set

$$
\begin{aligned}
\Theta_{m, k}(\zeta)= \begin{cases}\left(\prod_{i=1}^{N} \varphi_{i}(\zeta) \varphi_{i}\left(y_{m, k}\right)\right)^{1 / 8} & \text { if } k \leqslant k_{m}, \\
\frac{\left(\prod_{i=1}^{N} \varphi_{i}(\zeta) \varphi_{i}\left(y_{m, k}\right)\right)^{1 / 8}}{\varphi_{m_{R}}\left(y_{m, k}\right)^{1 / 4}-\varphi_{m_{R}}(\zeta)^{1 / 4}} & \text { if } k>k_{m}, \text { and }\end{cases} \\
\Lambda_{m, k}(\zeta)=\left(\Theta_{m, k}(\zeta)\right)^{-1} \Psi_{m, k, 0}(\zeta) .
\end{aligned}
$$

Estimating in the same manner as in the proof of Lemma 5.2 gives

$$
\left|\Theta_{m, k}(\zeta)\right| \approx \begin{cases}\inf _{(1 \leqslant i \leqslant N)}\left|\zeta-z_{i}\right|^{1 / 8} & \text { if } k \leqslant k_{m}, \\ \rho_{m}^{-\left|k-r_{m}\right| / 8} \inf _{\left(z_{i} \neq y_{m, \infty}\right)}\left|\zeta-z_{i}\right|^{1 / 8} & \text { if } k>k_{m},\end{cases}
$$

so that

$$
\left|\Lambda_{m, k}(\zeta)\right|< \begin{cases}c \inf _{(1 \leqslant i \leqslant N)}\left|\zeta-z_{i}\right|^{1 / 8} & \text { if } k \leqslant k_{m} \\ c \rho_{m}^{-\left|k-r_{m}\right| / 8} \inf _{\left(z_{i} \neq y_{m, \infty}\right)}\left|\zeta-z_{i}\right|^{1 / 8} & \text { if } k>k_{m} .\end{cases}
$$


Let $h \in H^{\infty}(\mathcal{O})$ and let $f, g \in L^{2}(\Omega)$. Then

$$
\begin{array}{r}
\langle h(T) f,\rangle=\left\langle h(T) \sum_{m, k} \Phi_{m, k, 0}(T) f, g\right\rangle \\
\leqslant \sup _{(m, k)}\left\|h(T) \Theta_{m, k}(T)\right\| \sum_{m, k}\left\|\Lambda_{m, k}(T) f\right\|\left\|\widetilde{\Psi}_{m, k, 0}\left(T^{*}\right) g\right\| \\
\leqslant \sup _{(m, k)}\left\|h(T) \Theta_{m, k}(T)\right\|\left(\int_{\Omega} \sum_{m, k}\left|\Lambda_{m, k}(T) f(x)\right|^{2} d x\right)^{1 / 2} \\
\cdot\left(\int_{\Omega} \sum_{m, k}\left|\widetilde{\Psi}_{m, k, 0}\left(T^{*}\right) g(x)\right|^{2} d x\right)^{1 / 2} .
\end{array}
$$

Using the randomisation lemma, (18), (19) and the fact that $T$ has a bounded $H^{\infty}\left(\mathcal{O}_{1}\right)$ functional calculus, one sees that the above is bounded by

$$
c \sup _{(m, k)}\left\|h(T) \Theta_{m, k}(T)\right\|\|f\|\|g\| .
$$

Further by (18) one has,

$$
\left\|h(T) \Theta_{m, k}(T)\right\| \leqslant c \int_{\partial \mathcal{O}}\left\|\frac{h(z) \Theta_{m, k}(z)}{z-T}\right\| \leqslant c \sup _{\zeta \in \mathcal{O}}|h(\zeta)|
$$

Thus $T$ has a bounded $H^{\infty}(\mathcal{O})$ functional calculus.

We close this section with the remark that the kernel $\mathcal{K}(z, \zeta)$ could be replaced by any other kernel which reproduces the value of holomorphic functions on $\mathcal{O}_{2}$ and is in $L^{1}\left(\partial \mathcal{O}_{2},|d \lambda|\right)$ uniformly for $\zeta \in \mathcal{O}_{1}$.

\section{Matricial functional Calculus on Hilbert space.}

Let $T$ be an operator acting on a complex Hilbert space $\mathcal{H}$ with $\sigma(T)$ contained in $\Delta$, which satisfies (16) and (17). Using the results and objects defined in Section 5 we consider holomorphic matrix-valued functions of $T$. We show that if $T$ has a bounded $H^{\infty}\left(\mathcal{O}_{1}\right)$ functional calculus then $T$ has a bounded $H^{\infty}\left(\mathcal{O}_{3} ; \mathbb{C}^{n \times n}\right)$ functional calculus, with bounds independent of the dimension $n$ (see also [14] and [8]).

First we note that if $\left[f_{r, s}\right] \in H^{\infty}\left(\mathcal{O}_{3} ; \mathbb{C}^{n \times n}\right)$ then, as an element of $H^{\infty}\left(\mathcal{O}_{1} ; \mathbb{C}^{n \times n}\right)$, one has the following decomposition:

$$
\left[f_{r, s}\right](\zeta)=\sum_{m, k, j} \Phi_{m, k, j}(\zeta)\left[\alpha_{m, k, j}^{r, s}\right]
$$

where the matrix $\left[\alpha_{m, k, j}^{r, s}\right]$ is given by the formula

$$
\left[\alpha_{m, k, j}^{r, s}\right]=\int_{\gamma_{m, k}} e_{m, k, j}(z)\left[f_{r, s}\right]|d \lambda|,
$$


and hence

$$
\left\|\left[\alpha_{m, k, j}^{r, s}\right]\right\|_{\infty} \leqslant c\left\|\left[f_{r, s}\right]\right\|_{\infty} .
$$

Let $(\mathcal{H})^{(n)}$ denote the space of $n$-tuples of vectors in $\mathcal{H}$. For $u \in(\mathcal{H})^{(n)}$ set

$$
\|u\|_{2,2}=c\left(\sum_{\ell=1}^{n}\left\|u_{\ell}\right\|^{2}\right)^{1 / 2} .
$$

With this definition we have the following theorem.

THEOREM 6.1. Let $T$ be an operator acting on a complex Hilbert space $\mathcal{H}$ which satisfies (16) and (17). Suppose $T$ has a bounded $H^{\infty}\left(\mathcal{O}_{1}\right)$ functional calculus. Then for $n=1,2, \ldots,\left[f_{r, s}(z)\right] \in H^{\infty}\left(\mathcal{O}_{3} ; \mathbb{C}^{n \times n}\right)$, and $u, v \in\left(L^{2}\right)^{(n)}$

$$
\left|\left\langle\left[f_{r, s}(T)\right] u, v\right\rangle\right| \leqslant c\left\|\left[f_{r, s}\right]\right\|_{\infty}\|u\|_{2,2}\|v\|_{2,2}
$$

where $c$ does not depend on $n$.

Proof: As in the proof of Theorem 5.5 we assume without loss of generality that $\mathcal{H}=L^{2}(\Omega)$. Fix $\left[f_{r, s}(z)\right] \in H^{\infty}\left(\mathcal{O}_{3} ; \mathbb{C}^{n \times n}\right)$ with $\left\|\left[f_{r, s}\right]\right\|_{\infty}=1$, and $u, v \in\left(L^{2}\right)^{(n)}$. Let $\langle\cdot, \cdot\rangle$ denote the bilinear pairing between $\left(L^{2}\right)^{(n)}$ and itself, and $\langle\cdot, \cdot\rangle_{\mathbb{C}^{n}}$ denote the bilinear pairing between $\mathbb{C}^{n}$ and itself. Decompose $\left[f_{r, s}\right]$ as in (20) so that

$$
\begin{aligned}
\left|\left\langle\left[f_{r, s}(T)\right] u, v\right\rangle\right| & \leqslant \sum_{m, k, j}\left|\left\langle\Phi_{m, k, j}(T)\left[\alpha_{m, k, j}^{r, s}\right] u, v\right\rangle\right| \\
& =\sum_{m, k, j}\left|\left\langle\Psi_{m, k, j}(T)\left[\alpha_{m, k, j}^{r, s}\right] u, \widetilde{\Psi}_{m, k, j}\left(T^{*}\right) v\right\rangle\right| \\
& \leqslant \int_{\Omega} \sum_{m, k, j}\left|\left\langle\dot{\Psi}_{m, k, j}(T)\left[\alpha_{m, k, j}^{r, s}\right] u(x), \tilde{\Psi}_{m, k, j}\left(T^{*}\right) v(x)\right\rangle_{\mathbb{C}^{n}}\right| .
\end{aligned}
$$

Now, the Cauchy-Schwarz inequality and Lemma 5.2 show that the above is

$$
\begin{aligned}
& \leqslant c \int_{\Omega} \sum_{m, k, j}\left(\sum_{\ell=1}^{n}\left|\Psi_{m, k, j}(T) u_{\ell}(x)\right|^{2}\right)^{1 / 2}\left(\sum_{\ell=1}^{n}\left|\tilde{\Psi}_{m, k, j}\left(T^{*}\right) v_{\ell}(x)\right|^{2}\right)^{1 / 2} \\
& \leqslant c\left(\sum_{\ell=1}^{n}\left\|\left(\sum_{m, k, j}\left|\Psi_{m, k, j}(T) u_{\ell}(\cdot)\right|^{2}\right)^{1 / 2}\right\|^{2}\right)^{1 / 2}\left(\sum_{\ell=1}^{n}\left\|\left(\sum_{m, k, j}\left|\widetilde{\Psi}_{m, k, j}\left(T^{*}\right) v_{\ell}(\cdot)\right|^{2}\right)^{1 / 2}\right\|^{2}\right)^{1 / 2} \\
& \leqslant c\|u\|_{2,2}\|v\|_{2,2} .
\end{aligned}
$$

Hence $T$ has a uniformly bounded matricial functional calculus.

We now discuss an interesting special case of Theorem 6.1. Let $\mathbb{D}$ denote the open unit disk in $\mathbb{C}$, and let $T$ be an operator acting on a Hilbert space which has a 
bounded $H^{\infty}(\mathbb{D})$ functional calculus. We also suppose that there exists finitely many points $z_{1}, z_{2}, \ldots, z_{n}$ contained in $\partial \mathbb{D}$ such that for $z$ outside $\mathbb{D}$,

$$
\left\|(z-T)^{-1}\right\| \leqslant c \sup _{i}\left|\left(z-z_{i}\right)^{-1}\right|
$$

It follows that there exists a closed set $\Delta$ contained in the closure of $\mathbb{D}$ which touches the boundary of the $\mathbb{D}$ nontangentially at $z_{1}, z_{2}, \ldots, z_{n}$ and for which (21) holds with a possibly larger constant. If $\tau<1$, then Theorems 5.3 and 6.1 show that $\tau T$ has a uniformly bounded matricial functional calculus on $\mathbb{D}$. Examination of their proofs shows that for $\left[f_{r, s}(z)\right] \in H^{\infty}\left(\mathbb{D} ; \mathbb{C}^{n \times n}\right)$,

$$
\sup _{\tau<1}\left\|\left[f_{r, s}(\tau T)\right]\right\| \leqslant c\left\|\left[f_{r, s}\right]\right\|_{\infty} .
$$

In [15], Paulsen showed that this implies that $T$ is similar to a contraction. We record the above observation in the following theorem stated in the language usual for this problem.

THEOREM 6.2. If $T$ is a polynomially bounded operator for which there exists finitely many points $z_{1}, z_{2}, \ldots, z_{n}$ contained in $\partial \mathbb{D}$ such that (21) holds, then $T$ is similar to a contraction.

An operator $T$ is polynomially bounded if for every polynomial $p,\|p(T)\| \leqslant \sup _{z \in \mathbb{D}}|p(z)|$.

\section{REFERENCES}

[1] D. Albrecht, Functional calculi of commuting unbounded operators, Ph.D. thesis (Monash University, Melbourne, Australia, 1994).

[2] D. Albrecht, X.T. Duong and A. McIntosh, 'Operator theory and harmonic analysis', in Workshop on Analysis and Geometry, 1995, Part III, Proceedings of the Centre for Mathematics and its Applications 34 (C.M.A., A.N.U., Canberra, Australia, 1996), pp. 77-136.

[3] D. Albrecht, E. Franks and A. McIntosh, Holomorphic functional calculi and sums of commuting operators (Macquarie University Mathematics Report, 1998).

[4] P. Auscher, A. McIntosh and A. Nahmod, 'Holomorphic functional calculi of operators, quadratic estimates and interpolation', Indiana Univ. Math. J. 46 (1997), 375-403.

[5] K. Boyadzhiev and R. de Laubenfels, 'Semigroups and resolvents of bounded variation, imaginary powers and $H^{\infty}$ functional calculus', Semigroup Forum 45 (1992), 372-384.

[6] M. Cowling, I. Doust, A. McIntosh and A. Yagi, 'Banach space operators with a bounded $H^{\infty}$ functional calculus', J. Austral. Math. Soc. Ser. A 60 (1996), 51-89.

[7] E. Franks, 'Polynomially subnormal operator tuples', J. Operator Theory 31 (1994), 219-228.

[8] E. Franks, 'Modified Cauchy kernels and functional calculus for operators on Banach space', J. Austral. Math. Soc. Ser. A 63 (1997), 91-99.

[9] E. Franks, 'A new approach to quadratic estimates, functional calculus, and similarity for type $\omega$ operators on Hilbert spaces', (in preparation). 
[10] F. Lancien, G. Lancien and C. Le Merdy, 'A joint functional calculus for sectorial operators with commuting resolvents', Proc. London Math. Soc. (to appear).

[11] G. Lancien and C. Le Merdy, 'A generalized $H^{\infty}$ functional calculus for operators on subspaces of $L^{p}$ and application to maximal regularity', (preprint).

[12] A. McIntosh and A. Yagi, 'Operators of type- $\omega$ without a bounded $H^{\infty}$ functional calculus', in Miniconference on Operators in Analysis, Proc. Centre. Math. Analysis 24 (A.N.U., Canberra, Australia, 1989), pp. 159-172.

[13] A. McIntosh, 'Operators which have an $H^{\infty}$ functional calculus', in Miniconference on Operator Theory and Partial Differential Equations, Proc. Centre Math. Analysis 14 (A.N.U., Canberra, Australia, 1986), pp. 210-231.

[14] C. Le Merdy, 'The similarity problem for bounded analytic semigroups on Hilbert space', Semigroup Forum 56 (1998), 205-224.

[15] V.I. Paulsen, 'Every completely polynomially bounded operator is similar to a contraction', J. Funct. Anal. 55 (1984), 1-17.

[16] E.M. Stein, Singular Integrals and Differentiability Properties of Functions (Princeton University Press, Princeton, New Jersey, 1970).

Department of Mathematics

Macquarie University

New South Wales 2109

Australia

e-mail: edwin@mpce.mq.edu.au

alan@mpce.mq.edu.au 